\title{
DECOMPOSITION METHOD FOR SOLVING A SYSTEM OF THIRD-ORDER BOUNDARY VALUE PROBLEMS
}

\author{
Muhammad Aslam Noor ${ }^{1}$, Khalida Inayat Noor ${ }^{1}$ and Asif Waheed $^{2}$ \\ ${ }^{1}$ Mathematics Department, COMSATS Institute of Information Technology, \\ Park Road, Islamabad, Pakistan \\ ${ }^{2}$ Mathematics Department, HITEC University, Texila Cantt, Islamabad, Pakistan \\ noormaslam@hotmail.com, khalidanoor@hotmail.com, waheedasif@hotmail.com
}

\begin{abstract}
In this paper, we use the modified decomposition method for solving the system of third-order nonlinear boundary value problems associated with obstacle problems. Some examples of system of third-order nonlinear boundary value problems are given. The comparison of the results obtained by modified decomposition method with modified variation of parameters method is also given. Results are also shown graphically that demonstrate the nature of the obstacles in particular problems. The results are very encouraging indicating the reliability and efficiency of the technique.
\end{abstract}

Keywords- Modified decomposition method, Adomian's polynomials, system of thirdorder nonlinear boundary value problem.

\section{INTROUCTION}

In recent years, much attention has been given to solve a system of third-order obstacle boundary value problems, see $[1-4,5,6,7]$. To be more precise, we consider the following systems of third-order nonlinear boundary value problems:

$$
u^{\prime \prime \prime}= \begin{cases}f(x, u(x)), & a \leq x<c, \\ f(x, u(x))+u(x) g(x)+r, & c \leq x<d, \\ f(x, u(x)), & d \leq x \leq b,\end{cases}
$$

and

$$
u^{\prime \prime \prime}= \begin{cases}f(x, u(x))+u(x) g(x)+r, & a \leq x<c, \\ f(x, u(x)), & c \leq x<d, \\ f(x, u(x))+u(x) g(x)+r, & d \leq x \leq b,\end{cases}
$$

with boundary conditions

$$
u(a)=\alpha_{1}, u^{\prime}(a)=\alpha_{2}, u(b)=\alpha_{3},
$$

and continuity conditions of $u(x), u^{\prime}(x)$ and $u^{\prime \prime}(x)$ at internal points $c$ and $d$ of the interval $[a, b]$. Here $r$ and $\alpha_{i}, i=1 \ldots 3$ are real and finite constants and $g(x)$ is a continuous function on $[a, b]$. For simplicity, we will consider $f(x, u(x))=f(u)$. Systems (1a) and (1b) arise in obstacle, contact, unilateral and equilibrium problems. 
Most of the problems in oceanography, economics, transportation, nonlinear optimization, ocean wave engineering, fluid flow through porous media and some other branches of science and engineering are modeled by these systems, see [1-11] and references therein. Several techniques have been used to solve system of linear thirdorder boundary value problems associated with obstacle, contact and unilateral problems. All the above mentioned methods are proposed to solve linear system of boundary value problems associated with obstacle, contact and unilateral problems. Noor et al. [5] have proposed modified variation of parameters method for solving system of nonlinear obstacle boundary value problems of even and odd orders. Inspired and motivated by the recent research activities in this field, we consider the systems of nonlinear boundary value problems.

Adomian [12] developed a novel technique known as Adomian decomposition method for solving boundary value problems. Further Wazwaz [13] has modified this technique known as modified decomposition method. Modified decomposition method has been extensively used to solve a class of problems of diversified nature. In this paper, we again use the modified decomposition method to solve systems of thirdorder nonlinear obstacle boundary value problems. The modified decomposition method provides the rapidly convergent series solution with easily computable components. This technique makes the solution procedure simple while still maintaining the higher level of accuracy. In the present study, we implement this technique for solving systems of third-order nonlinear boundary value problems. The idea and technique of this paper may lead to novel applications of these problems in pure and applied sciences.

\section{MODIFIED DECOMPOSITION METHOD}

Consider the following differential equation:

$L(u)+R(u)+N(u)=g$,

where $L$ is the highest-order derivative which is assumed to be invertible, $R$ is a linear differential operator of order lesser than $L, N u$ represents the nonlinear terms and $g$ is the source term. Applying the inverse operator $L^{-1}$ to both sides of (2) and using the given conditions, we obtain $u=f-L^{-1}(R u)-L^{-1}(N u)$, where the function $f$ represents the terms arising from integrating the source term $g$ and by using the given conditions. In modified decomposition method [13], the function $f$ can be set as the sum of two partial functions namely $f_{1}$ and $f_{2}$ and defines the solution $u(x)$ by the series

$$
u(x)=\sum_{n=0}^{\infty} u_{n}(x)
$$

where the components $u_{n}(x)$ are usually determined recurrently by using the relation

$$
\begin{aligned}
& u_{0}=f_{1}, \quad u_{1}=f_{2}+L^{-1}\left(R u_{0}\right)-L^{-1}\left(N u_{0}\right), \\
& u_{k+1}=f_{2}+L^{-1}\left(R u_{k}\right)-L^{-1}\left(N u_{k}\right), \quad k \geq 1 .
\end{aligned}
$$

The nonlinear operator $N(u)$ can be decomposed into an infinite series of polynomials given by 


$$
N(u)=\sum_{n=0}^{\infty} A_{n},
$$

where $A_{n}$ are the so-called Adomian's polynomials. These Adominan's polynomials can be generated for various classes of nonlinearities according to the specific algorithm developed in [13] which yields

$$
A_{n}=\left(\frac{1}{n !}\right)\left(\frac{d^{n}}{d \lambda^{n}}\right) N\left(\sum_{i=0}^{n}\left(\lambda^{i} u_{i}\right)\right)_{\lambda=0}, n=0,1,2, \cdots
$$

\section{NUMERICAL RESULTS}

In this section, we apply the modified decomposition method [13] for solving a system of third-order nonlinear obstacle boundary value problems. The solutions are compared with modified variation of parameters method and are also graphically represented. The results are very encouraging indicating the reliability and efficiency of the technique.

Example 3.1 . Consider the system of third-order nonlinear boundary value problems equivalent to system (1a), $f(u)=\frac{u^{3}}{3 !}+\frac{u^{2}}{2 !}+u+1, g=1, r=-1$ and $[a, b]=[-1,1]$, as follows:

$$
u^{\prime \prime \prime}= \begin{cases}\frac{u^{3}}{3 !}+\frac{u^{2}}{2 !}+u+1, & \text { for }-1 \leq x<-\frac{1}{2} \\ \frac{u^{3}}{3 !}+\frac{u^{2}}{2 !}+2 u, & \text { for }-\frac{1}{2} \leq x<\frac{1}{2}, \\ \frac{u^{3}}{3 !}+\frac{u^{2}}{2 !}+u+1, & \text { for } \frac{1}{2} \leq x \leq 1,\end{cases}
$$

with boundary conditions $\quad u(-1)=u(1)=0, u^{\prime}(-1)=1$.

Case 1: $-1 \leq x<-\frac{1}{2}$. In this case, we implement modified decomposition method as follows

$$
\begin{aligned}
& L u=\frac{u^{3}}{3 !}+\frac{u^{2}}{2 !}+u+1 . \quad \text { Applying } L^{-1} \text { on both the sides, we have } \\
& u(x)=c_{3}+c_{2} x+c_{1} \frac{x^{2}}{2 !}+L^{-1}\left(\frac{u^{3}}{3 !}+\frac{u^{2}}{2 !}\right)+L^{-1}(u+1),
\end{aligned}
$$

where the operator $L^{-1}$ is defined as follows: $L^{-1}=\int_{0}^{x} \int_{0}^{x} \int_{0}^{x}() d x d x d$.$x , and c_{1}, c_{2}$ and $c_{3}$ will be determined further by using boundary conditions and continuity conditions. 
Substituting the decomposition series (3) for $u(x)$ and the series of polynomials for nonlinearity (4) into (6), we have

$$
\sum_{n=0}^{\infty} u_{n}=c_{3}+c_{2} x+c_{1} \frac{x^{2}}{2}+L^{-1}\left(\sum_{n=0}^{\infty} A_{n}\right)+L^{-1}\left(\sum_{n=0}^{\infty} u_{n}+1\right),
$$

Using the modified decomposition method [13], we have following approximations

$$
\begin{aligned}
u_{0}(x) & =c_{2} x \\
u_{1}(x) & =c_{3}+\frac{1}{2} c_{1} x^{2}+\frac{1}{6} x^{3}+\frac{1}{24} c_{2} x^{4}+\frac{1}{120} c_{2}{ }^{2} x^{5}+\frac{1}{720} c_{2}{ }^{3} x^{6}, \\
u_{2}(x) & =\frac{1}{6} c_{3} x^{3}+\frac{1}{24} c_{2} c_{3} x^{4}+\left(\frac{1}{120} c_{1}+\frac{1}{120} c_{2}{ }^{2} c_{3}\right) x^{5}+\left(\frac{1}{720}+\frac{1}{240} c_{1} c_{2}\right) x^{6} \\
& +\left(\frac{1}{1008} c_{2}+\frac{1}{840} c_{1} c_{2}{ }^{2}\right) c_{2} x^{7}+\frac{1}{2520} c_{2}{ }^{2} x^{8}+\frac{11}{181440} c_{2}{ }^{3} x^{9}+\ldots . .
\end{aligned}
$$

Case 1.1: $-\frac{1}{2} \leq x<\frac{1}{2}$. In this case, we have $L u=\frac{u^{3}}{3 !}+\frac{u^{2}}{2 !}+2 u$. Applying $L^{-1}$ on both the sides, we have

$$
u(x)=c_{6}+c_{5} x+c_{4} \frac{x^{2}}{2}+L^{-1}\left(\frac{u^{3}}{3 !}+\frac{u^{2}}{2 !}\right)+2 L^{-1}(u),
$$

Substituting the decomposition series for $u(x)$ and the series of polynomials for nonlinearity into (7), we have following approximations

$$
\begin{aligned}
u_{0}(x) & =c_{5} x \\
u_{1}(x) & =c_{6}+\frac{1}{2} c_{4} x^{2}+\frac{1}{12} c_{5} x^{4}+\frac{1}{120} c_{5}{ }^{2} x^{5}+\frac{1}{720} c_{5}^{3} x^{6}, \\
u_{2}(x) & =\frac{1}{3} c_{6} x^{3}+\frac{1}{24} c_{5} c_{6} x^{4}+\left(\frac{1}{60} c_{4}+\frac{1}{120} c_{5}{ }^{2} c_{6}\right) x^{5}+\frac{1}{240} c_{4} c_{5} x^{6}+\left(\frac{1}{1260} c_{5}+\frac{1}{840} c_{5}{ }^{2} c_{4}\right) c_{2} x^{7} \\
& +\frac{1}{3360} c_{5}{ }^{2} x^{8}+\frac{19}{181440} c_{5}^{3} x^{9}+\frac{1}{129600} c_{5}^{4} x^{10}+\frac{1}{1425600} c_{5}{ }^{5} x^{11},
\end{aligned}
$$

Case 1.1.1: $\frac{1}{2} \leq x \leq 1$. In this case, we have $L u=\frac{u^{3}}{3 !}+\frac{u^{2}}{2 !}+u+1$. Applying $L^{-1}$ on both the sides, we have

$$
u(x)=c_{9}+c_{8} x+c_{7} \frac{x^{2}}{2}+L^{-1}\left(\frac{u^{3}}{3 !}+\frac{u^{2}}{2 !}\right)+L^{-1}(u+1),
$$


Substituting the decomposition series for $u(x)$ and the series of polynomials for nonlinearity into (8), we have following approximations

$$
\begin{aligned}
u_{0}(x) & =c_{8} x \\
u_{1}(x) & =c_{9}+\frac{1}{2} c_{7} x^{2}+\frac{1}{6} x^{3}+\frac{1}{24} c_{8} x^{4}+\frac{1}{120} c_{8}{ }^{2} x^{5}+\frac{1}{720} c_{8}{ }^{3} x^{6}, \\
u_{2}(x) & =\frac{1}{6} c_{9} x^{3}+\frac{1}{24} c_{8} c_{9} x^{4}+\left(\frac{1}{120} c_{7}+\frac{1}{120} c_{8}{ }^{2} c_{9}\right) x^{5} \\
+ & \left(\frac{1}{720}+\frac{1}{240} c_{7} c_{8}\right) x^{6}+\left(\frac{1}{1008}+\frac{1}{840} c_{7} c_{8}\right) c_{8} x^{7} \\
& +\frac{1}{2520} c_{8}{ }^{2} x^{8}+\frac{11}{181440} c_{8}{ }^{3} x^{9}+\frac{1}{129600} c_{8}{ }^{4} x^{10}+\frac{1}{1425600} c_{8}{ }^{5} x^{11},
\end{aligned}
$$

By using modified decomposition method, we have following formula for getting series solution in the whole domain from the above cases

$$
u(x)=\left\{\begin{array}{lc}
\sum_{k=0}^{\infty} u_{k}(x) & \text { for }-1 \leq x \leq-\frac{1}{2}, \\
\sum_{k=0}^{\infty} u_{k}(x) & \text { for }-\frac{1}{2} \leq x \leq \frac{1}{2}, \\
\sum_{k=0}^{\infty} u_{k}(x) & \text { for } \frac{1}{2} \leq x \leq 1 .
\end{array}\right.
$$

Hence, we have the following series solution after two iterations 


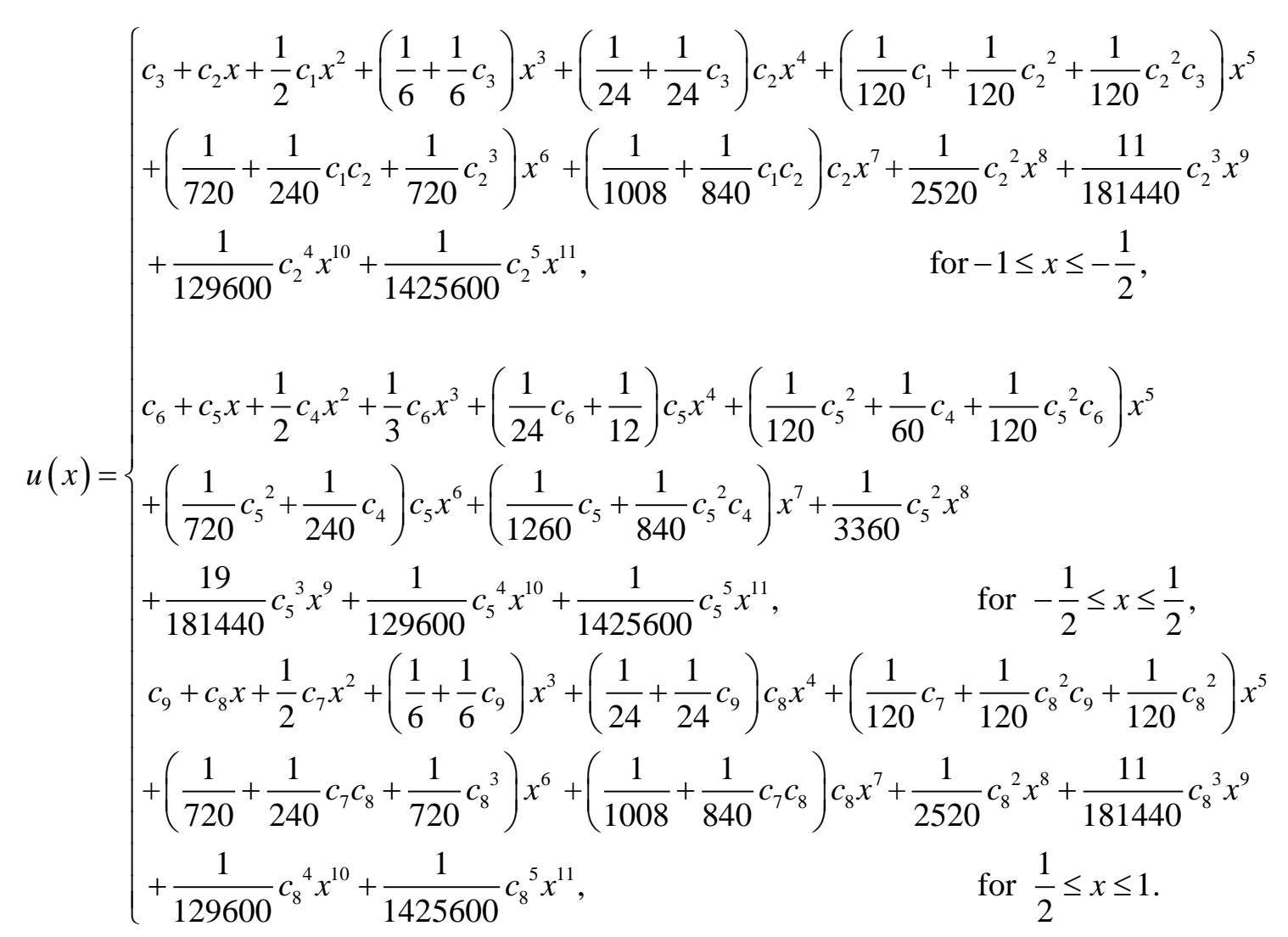

Now we use boundary conditions and continuity conditions at $x=-\frac{1}{2}$ and $x=\frac{1}{2}$, and we obtain system of nonlinear equations. In order to solve system of nonlinear equations, we use Newton's method. Hence we have the following values of unknown constants

$$
\begin{aligned}
& c_{1}=.6074700134, \mathrm{c}_{2}=.1210442317, \mathrm{c}_{3}=-.0208242536, c_{4}=.0860686116, \\
& \mathrm{c}_{5}=-.0086174581, \mathrm{c}_{6}=-.0423844106, \mathrm{c}_{7}=-.4306744635, \mathrm{c}_{8}=.1207451206, \\
& \mathrm{c}_{9}=-.0639441701 .
\end{aligned}
$$

By using values of unknowns from (10) into (9), we have following analytic solution of system of second-order nonlinear boundary value problems (5) 
236 Decomposition Method for Solving a System of Boundary Value Problems

$$
u(x)= \begin{cases}-.0208242536 .1210442317 x+.3037350067 x^{2}+.1631959578 x^{3}+.004938482330 x^{4} \\ +.005181805072 x^{5}+.001697730177 x^{6}+.0001306793633 x^{7}+5.814169060 \times 10^{-6} x^{8} \\ +1.075206652 \times 10^{-7} x^{9}+1.656423530 \times 10^{-9} x^{10}+1.822731942 \times 10^{-11} x^{11}, & \text { for }-1 \leq x<-\frac{1}{2}, \\ -.0423844106-.0086174581 x+.04303430580 x^{2}-.01412813687 x^{3}-.0007029029299 x^{4} & \\ +.001435069469 x^{5}-3.091274861 \times 10^{-6} x^{6}-6.831643525 \times 10^{-6} x^{7}+2.210136432 \times 10^{-8} x^{8} & \text { for }-\frac{1}{2} \leq x<\frac{1}{2}, \\ -6.701285256 \times 10^{-11} x^{9}+4.255119099 \times 10^{-14} x^{10}-3.333482776 \times 10^{-17} x^{11}, & \\ -.0639441701+.1207451206 x-.2153372318 x^{2}+.1560093050 x^{3}+.004709340587 x^{4} & \\ -.003475227883 x^{5}+.001174659541 x^{6}+.0001123118636 x^{7}+5.785469901 \times 10^{-6} x^{8} & \\ +1.067255537 \times 10^{-7} x^{9}+1.640111437 \times 10^{-9} x^{10}+1.800322301 \times 10^{-11} x^{11}, & \text { for } \frac{1}{2} \leq x \leq 1 .\end{cases}
$$

Table 3.1 shows the comparison of the analytic solution of problems (5) between modified decomposition method (MDM) and modified variation of parameters method(MVPM)

\begin{tabular}{|c|c|c|c|}
\hline Sr. No & MDM & MVPM & Error \\
\hline-1 & 0 & 0 & 0 \\
\hline-.8 & -.0060821310 & -.0060821310 & $8.00 \mathrm{E}-10$ \\
\hline-.6 & -.0190437794 & -.0190437793 & $1.40 \mathrm{E}-10$ \\
\hline-.4 & -.0311804287 & -.0311804286 & $1.10 \mathrm{E}-10$ \\
\hline-.2 & -.0388281058 & -.0388281056 & $2.00 \mathrm{E}-10$ \\
\hline 0 & -.0423844109 & -.0423844106 & $3.00 \mathrm{E}-10$ \\
\hline .2 & -.0425002212 & -.0425002208 & $4.20 \mathrm{E}-10$ \\
\hline .4 & -.0398534293 & -.0398534287 & $6.10 \mathrm{E}-10$ \\
\hline .6 & -.0349223481 & -.0349223473 & $8.00 \mathrm{E}-10$ \\
\hline .8 & -.0241644871 & -.0241644860 & $1.02 \mathrm{E}-10$ \\
\hline 1 & 0 & 0 & 0 \\
\hline
\end{tabular}




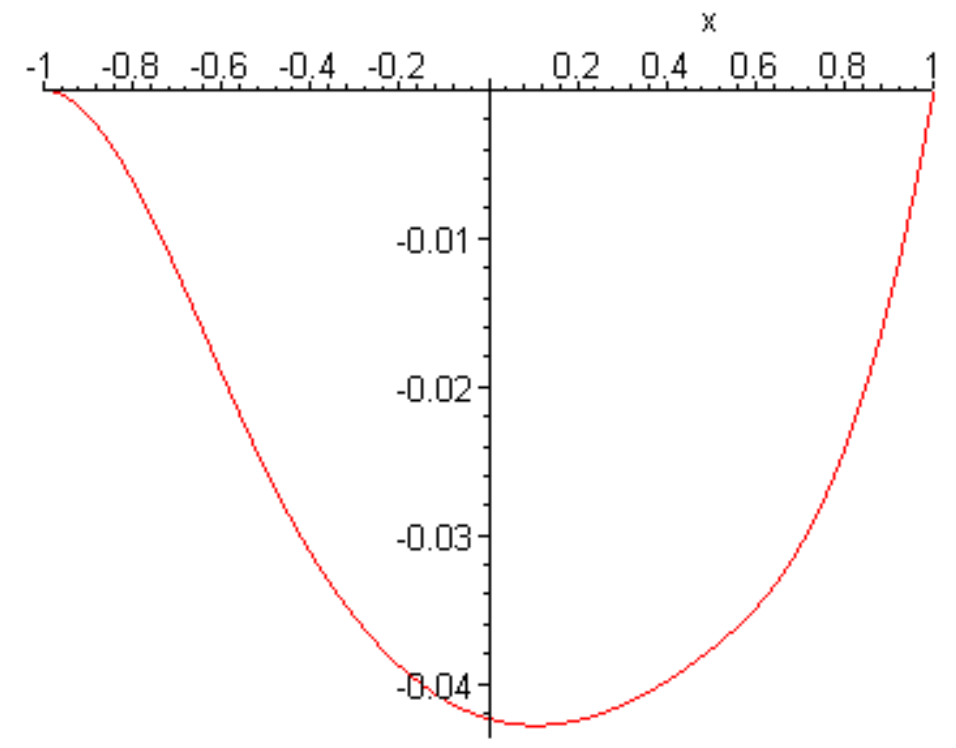

Figure 3.1 depicts the graphical representation of analytical solution of (5) by using modified decomposition method

Example 3. 2. Consider the system of third-order nonlinear boundary value problems equivalent to system (1b), $f(u)=2 u^{3}, g=1, r=-1$ and $[a, b]=[-1,1]$, as follows:

$$
u^{\prime \prime \prime}= \begin{cases}-1+u+2 u^{3}, & \text { for }-1 \leq x<-\frac{1}{2}, \\ 2 u^{3}, & \text { for }-\frac{1}{2} \leq x<\frac{1}{2}, \\ -1+u+2 u^{3}, & \text { for } \frac{1}{2} \leq x \leq 1,\end{cases}
$$

with boundary conditions $\quad u(-1)=u(1)=0, u^{\prime}(-1)=1$.

Using the procedure of Example 3.1, we have the following series solution after two iterations 


$$
u(x)=\left\{\begin{array}{l}
c_{3}+c_{2} x+\frac{1}{2} c_{1} x^{2}+\left(\frac{1}{6} c_{3}-\frac{1}{6}\right) x^{3}+\frac{1}{24} c_{2} x^{4}+\left(\frac{1}{120} c_{1}+\frac{1}{10} c_{2}{ }^{2} c_{3}\right) x^{5}+\left(\frac{1}{60} c_{2}^{3}-\frac{1}{720}\right) x^{6} \\
+\left(\frac{1}{70} c_{2} c_{1}+\frac{1}{5040}\right) c_{2} x^{7}-\frac{1}{336} c_{2}{ }^{2} x^{8}+\frac{1}{1890} c_{2}^{3} x^{9}+\frac{1}{9900} c_{2}^{5} x^{11}, \quad \text { for }-1 \leq x<-\frac{1}{2} \\
c_{6}+c_{5} x+\frac{1}{2} c_{4} x^{2}+\frac{1}{10} c_{5}{ }^{2} c_{6} x^{5}+\frac{1}{60} c_{5}^{3} x^{6}+\frac{1}{70} c_{5}{ }^{2} c_{4} x^{7}+\frac{1}{9900} c_{5}^{5} x^{11}, \quad \text { for }-\frac{1}{2} \leq x<\frac{1}{2} \\
c_{9}+c_{8} x+\frac{1}{2} c_{7} x^{2}+\left(\frac{1}{6} c_{9}-\frac{1}{6}\right) x^{3}+\frac{1}{24} c_{8} x^{4}+\left(\frac{1}{120} c_{7}+\frac{1}{10} c_{8}^{2} c_{9}\right) x^{5}+\left(\frac{1}{60} c_{8}{ }^{3}-\frac{1}{720}\right) x^{6} \\
+\left(\frac{1}{70} c_{8} c_{7}+\frac{1}{5040}\right) c_{8} x^{7}-\frac{1}{336} c_{8}{ }^{2} x^{8}+\frac{1}{1890} c_{8}^{3} x^{9}+\frac{1}{9900} c_{8}^{5} x^{11}, \quad \text { for } \frac{1}{2} \leq x \leq 1 .
\end{array}\right.
$$

Now we use boundary conditions and continuity conditions at $x=-\frac{1}{2}$ and $x=\frac{1}{2}$, and we obtain system of nonlinear equations. Hence we have the following values of unknown constants by using the Newton method.

$$
\begin{aligned}
& c_{1}=-1.3277592790, \mathrm{c}_{2}=-.0523372238, \mathrm{c}_{3}=.5249671413, c_{4}=-1.0719942040, \\
& \mathrm{c}_{5}=.0140953602, \mathrm{c}_{6}=.5362933240, \mathrm{c}_{7}=-.8198949226, \mathrm{c}_{8}=-.0519640849, \\
& \mathrm{c}_{9}=.5476022141 .
\end{aligned}
$$

By using values of unknowns from (16) into (15), we have following analytic solution of system of second-order nonlinear boundary value problems (11)

$$
u(x)=\left\{\begin{array}{lr}
.5249671413-.0523372238 x-.6638796395 x^{2}-.07917214315 x^{3}-.002180717658 x^{4} \\
-.01092086245 x^{5}-.001391278245 x^{6}-6.234120257 \times 10^{-5} x^{7}-8.152336295 \times 10^{-6} x^{8} \\
-7.585255984 \times 10^{-8} x^{9}-3.966598244 \times 10^{-11} x^{11}, & \text { for }-1 \leq x<-\frac{1}{2}, \\
.5362933240+.0140953602 x-.5359971020 x^{2}+1.065503174 \times 10^{-5} x^{5} & \\
+4.667424325 \times 10^{-8} x^{6}-3.042613266 \times 10^{-6} x^{7}+5.620121416 \times 10^{-14} x^{11}, & \text { for }-\frac{1}{2} \leq x<\frac{1}{2} \\
.5476022141-.0519640849 x-.4099474613 x^{2}-.07539963101 x^{3}-.002165170204 x^{4} \\
-.006684590518 x^{5}-.001391227503 x^{6}-4.193796975 \times 10^{-5} x^{7}-8.036506307 \times 10^{-6} x^{8}
\end{array}\right.
$$


Table 3.2 represents the comparison of the analytic solution of problems (11) between modified decomposition method (MDM) [11] and modified variation of parameters method (MVPM) [5]

\begin{tabular}{|c|c|c|c|}
\hline Sr. No & MDM & MVPM & Error \\
\hline-1 & 0 & 0 & 0 \\
\hline-.8 & .1848224157 & .1848224157 & 0 \\
\hline-.6 & .3349772712 & .3349772711 & $1.00 \mathrm{E}-10$ \\
\hline-.4 & .4448955397 & .4448955397 & 0 \\
\hline-.2 & .5120343645 & .5120343646 & $1.00 \mathrm{E}-10$ \\
\hline 0 & .5362933240 & .5362933242 & $2.00 \mathrm{E}-10$ \\
\hline .2 & .5176725153 & .5176725156 & $3.00 \mathrm{E}-10$ \\
\hline .4 & .4561720361 & .4561720363 & $2.00 \mathrm{E}-10$ \\
\hline .6 & .3516897381 & .3516897382 & $1.00 \mathrm{E}-10$ \\
\hline .8 & .2016078444 & .2016078444 & 0 \\
\hline 1 & 0 & 0 & 0 \\
\hline
\end{tabular}

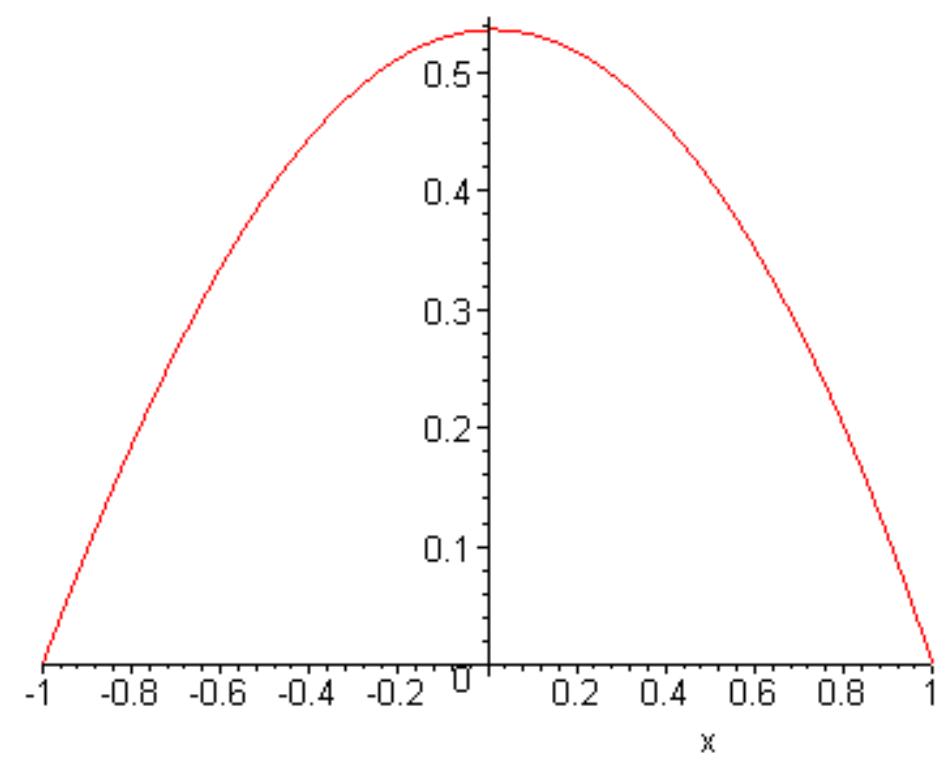

Figure 3.2 is a graphical representation of analytical solution of system of third-order nonlinear boundary value problem (11) by using modified decomposition method

\section{CONCLUDING REMARKS}

In this paper, we have used the modified decomposition method for solving system of third-order nonlinear boundary value problems. We have considered two examples for both the systems. The comparison of the results obtained by this method are also given with modified variation of parameters method. The results are also represented graphically which demonstrate the nature of the obstacle in each problem. It 
is an interesting problem to compare these methods with other methods such as variational iteration methods and its various modifications.

Acknlowdegement- The authors are grateful to Dr. S. M. Junaid Zaidi, Rector, COMSATS Institute of Information Technology, Pakistan for providing excellent research and academic environments. This research is partially supported by HEC NRPU project No: 20-1966/R \\&D/11-2553, titled: Research unit of Academic Excellence in Geometric Function Theory and Applications.. The authors would like to thank the referees for their valuable and constructive comments.

\section{REFERENCES}

1. F. Gao and C. M. Chi, Solving third-order obstacle problems with quartic B-splines, Applied Mathematics and Computation 180, 270-270, 2006.

2. A. K. Khalifa and M. A. Noor, Quintic splines solutions of a class of contact problems, Mathematical and Computer Modelling 13, 51-58, 1990.

3. S. Momani, K. Moadi and M. A. Noor, Decomposition method for solving system of forth-order obstacle boundary value problems, Applied Mathematics and Computation 175, 823-931, 2006.

4. M. A. Noor, Variational inequalities in physical oceanography, in: Ocean Wave Engineering (edit. M. Rahman), Computational Mechanics Publications, Southampton, UK, 201-226, 1994.

5. M. A. Noor, K. I. Noor, A. Waheed and E. A. Al-Said, Modified variation of parameters method for solving system of second-order nonlinear boundary value problem, International Journal of Physical Sciences 5, 2426-2431, 2010.

6. M. A. Noor and S. I. Tirmizi, Numerical methods for unilateral problems, Journal of Computational and Applied Mathematics 16, 387-395, 1986.

7. M. A. Noor and S. I. Trimizi, Finite difference techniques for solving obstacle problems, Applied Mathematics Letters 1, 267-271, 1988.

8. M. A. Noor, Some developments in general variational inequalities, Applied Mathematics and Computation 152, 199-277, 2004.

9. M. A. Noor and A. K. Khalifa, A numerical approach for odd-order obstacle problems, International Journal of Computer Mathematics 54, 109-116, 1994.

10. M. A. Noor, K. I. Noor and E. Al-Said, Iterative methods for solving nonconvex equilibrium variational inequalities, Applied Mathematics \& Information Sciences 6(1), 65-69, 2012.

11. S. Islam, M. A. Khan, I. A. Tirmizi, and E. H. Twizell, Non-polynomial spline approach to the solution of a system of third-order boundary-value problems, Applied Mathematics and Computation 168, 152-163, 2005.

12. G. Adomain, A review of the decomposition method in applied mathematics, Journal of Mathematical Analysis and Applications 13, 501-544, 1988.

13. A. M. Wazwaz, Approximate solutions to boundary value problems of higher-order by the modified decomposition method, Computers \& Mathematics with Applications 40, 679-691, 2000. 